\title{
KURZE BESCHREIBUNG EINES ELEKTRISCH REGISTRIERENDEN STROMMESSERS
}

voN

\section{ROLF J. WITTING}

Bei der Zusammenkunft von schwedischen und finnischen HydroB graphen, die im November 1903 in Nynäs stattfand, hatte ich die Gelegenheit, Prof. O. Petrersson's Bifilarstrommesser kennen zu lernen. Als ich dann im Frühling 1904 einen Strommesser für die finnischen Terminfahrten konstruierte, kam das bifilare Aufhängungssystem zur Anwendung. Da möglichst grosse Einfachheit erstrebt wurde, wurden Auslösung und Schliessung des Zählwerkes durch Fallgewichte vollzogen.

Die zeitraubende Arbeitsart - das Aufholen und Niederlassen des Apparats bei jeder neuen Tiefe - war entschieden ein Uebelstand, so auch die Knappheit der erhaltenen Angaben. Da die Zeit eine Wiederholung der Observationen nur selten gestattete, konnten nur eine Geschwindigkeits- und eine Richtungsangabe für jede Tiefe erhalten werden. Dass die Bewegungen des Schiffes nicht gemessen wurden, machte den Uebelstand um so fühlbarer.

Durch die Konstruktion des neuen unten beschriebenen Apparats sollten diese Uebel beseitigt werden. Darum wurde für den Strommesser eine Form erstrebt, die eine fortlaufende Beobachtungsreihe ermöglichte, und die es gestattete, den Apparat ohne jegliches Aufholen successiv in die verschiedenen Tiefen zu senken.

\section{Der Apparat}

Der Apparat besteht aus dem an einem bifilar aufgehängten $3 \mathrm{~m}$ langen Rahmen befestigten Strommesser und dem an Bord des arbeitenden Dampfers befindlichen Registrierungsapparate. Die Registrierung geschieht elektrisch. Von dem Registrierungsapparate führt ein Kabel aus 10 Leitungsdrähten zum Strommesser hinunter. Auf jeden zehnten Schlag des Stromflügels erfolgt die Schliessung eines galvanischen Stromkreises, und je nach der Richtung, in welche die Stromfahne vom Strome eingestellt wird, werden verschiedene andere zum Registrierungsapparate führende Leitungsbahnen geschlossen. 
Der Strommesser ist mittels der mit Klemmen versehenen Griffe $X$ und $Y$ am Rahmen befestigt. Ausser diesen Griffen sind die sie verbindende Vertikalachse und die an der letzteren befestigte Ebonitplatte $P$ unbeweglich im Verhältnis zum Rahmen; alle die übrigen Teile sind mittels 2 Kugelgelenke um die Achse beweglich.

Der achtblätterige Propeller hat an der Achse eine Schraube olme Ende, welche in das Zalınrad $H$ greift. Bei jedem zehnten Schlag gerät einer der kleinen Zapfen des Rades mit dem Kontakte $K$ in Berührung und schliesst dadurch die Schlagzählerleitung. Die gemeinsame Zufuhrleitung, die an die Klemme $Z$ gekoppelt ist, steht mit dem Rade $H$ durch die Vertikalachse und das Propellerhaus in unmittelbarer Verbindung.

Von dem Kontakte $K$ geht die Leitung, die während der Arbeit geschlossen bleiben muss, durch die von Ebonit umgebenen isolierten Stücke $S$ in das cylinderringformige mit Quecksilber gefüllte Ebonitgefäss $B$ und weiter durch den ins Quecksilber hineinragenden Stab St zur Klemme $G$ und schliesslich zum Schlagzählerwerke.

Am unteren Rande der Ebonitplatte $P$ sind 8 voneinander isolierte, einen Zirkel bildende Metallstücke befestigt, von denen ein jedes mittels einer Klemme mit einem Rückleitungsdrahte in Verbindung steht. An dem beweglichen Teil des Strommessers ist der Wagen $W$ so befestigt, dass er sich bei einer Drehung des Strommessers längs dem von den 8 Metallstücken gebildeten Zirkelring bewegt. Der Wagen steht in unmittelbarer metallischer Verbindung mit der Klemme $Z$, also mit der Zufulırleitung. Je nach der Lage des Strommessers wird der Wagen gegen bestimmte Metallstücke ruhen und werden bestimmte Leitungsbahnen zwischen dem Strommesser und dem Registrierungsapparate geschlossen.

Der Wagen hat zwei Räder, deren Tangierungspunkte mit den Metallstücken 1/16 des erwähnten Zirkels ausmachen ${ }^{1}$ ). Solange die Mitte des Wagens sich innerhalb der zwei mittleren Viertel des ${ }^{1 / 8}$ der Peripherie umfassenden Metallstückes befindet, berührt der Wagen nur dieses Stück; gleitet aber die Mitte auf eins der äusseren Viertel über, berührt jedes der Räder ein Metallstück und werden also zwei Leitungen geschlossen. So gibt der Apparat über 16 verschiedene Richtungen Auskunft, von denen jede einem Sektor von $22^{\circ} 5$ entspricht.

Da durch die Bewegungen des Fahrzeuges der Winkel zwischen dessen Längenrichtung und dem Strom wechseln kann und die Angaben des Registrierungsapparats sich also je nachdem verändern, kann durch Schätzung noch grössere Genauigkeit erlangt werden.

Ausser den stählernen Kugeln in den Kugellagern, der in Stahl-

1) Das Vorbild dieser Einrichtung findet sich an einer auf der hiesigen Meteorologischen Centralanstalt aufgestellten von Herrn Direktor E. Biese konstruierten registrierenden Windfahne. 
lagern beweglichen Propellerachse und der Stromfahne aus Aluminium sind sämtliche Teile des Strommessers aus Ebonit oder vernickeltem Messing. Von der c. $70 \mathrm{~cm}$ langen Stromfahne sind die letzten $20 \mathrm{~cm}$ gespalten mit einem Winkel von $20^{\circ}$ zwischen den Flügeln. Das Gegengewicht $\boldsymbol{E}$ equilibriert den Strommesser.

In der unteren rechten Ecke der Zeichnung sielst man den Registrierungsapparat im Durchschnitt. Dessen wichtigste Teile sind der Cylinder $C$, der von einem Uhrwerke in konstante Rotation $2.8 \mathrm{~mm} / \mathrm{sec}$ an der Peripherie gebracht wird, und über den das Registrierungspapier gleitet, und die Federn $F$, die durch Elektromagnete getrieben gegen dieses spielen. Jede Feder ist an einem um das Lager $A x$ beweglichen Arm befestigt, der durch die von der Schraube $O$ justierten Feder vom Cylinder weggehalten wird. Wenn ein elektrischer Strom die Windungen der Elektromagneten $U$ passiert, wird der untere aus weichem Eisen bestehende Teil des Armes gegen diese gezogen und die Registrierungsfeder wird zum Papiere geführt. Die erste Feder rechts steht mit ihrem besonderen Elemente in Verbindung und macht auf dem Papier äquidistante Zeichen, wenn der Kontakt $J$ die kleinen Zapfen des Cylinders $C$ berührt. Zum Elektromagneten der folgenden Feder ist die Schlagzählerleitung geführt und zu den übrigen der Reihe nach die Leitungen von den Richtungskontaktstücken.

Das für den Strommesser bestellte Kabel misst $9 \mathrm{~mm}$ im Diameter und enthält 10 Leitungsdrähte. Der verspäteten Lieferung zufolge musste aber bei der Augustexpedition ein provisorisches aus 10 gewöhnlichen, gegen Feuchtigkeit isolierten Leitungsdrähten gemachtes Kabel mit einem Diameter von $1.5 \mathrm{~cm}$ benutzt werden. Das Kabel war dicht am Strommesser befestigt. Die Winde, an welcher das Kabel aufgerollt wird, ist am einen Ende ihrer Achse mit 10 voneinander durch $\%$ wischenliegende Ebonitringe isolierten Messingringen versehıen. $\mathrm{Zu}$ diesen 10 Ringen sind die verschiedenen Drähte des Kabels durch die Achse geleitet worden, und von den gegen die Ringe friktierenden Gleitfedern ist die Leitung fortgesetzt.

Der Propeller, der keine Arretierungseinrichtung hat, ist nebst dem Kontakte $K$ und dem Rade $H$ am Rahmen $R$ befestigt. Dieser, zwischen zwei Schienen in den Strommesser eingeschoben, kann, nachdem die Schraube $J$ losgemacht worden ist, herausgenommen werden und ist als der empfindlichste Teil des Apparats mit grösster Vorsicht zu verwahren.

Der Strommesser und der Registrierungsapparat sind bei dem Statsmechaniker V. Falck-Rasmussen in Helsingfors verfertigt worden. Das Uhrwerk hat Richard, Paris, geliefert. 


\section{Etwaige Veränderungen}

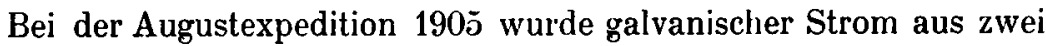
Trockenelementen angewendet. Wie es ja auch zu erwarten war, trat Polarisation ein. Eine Reinigung nach jeder oder jeder zweiten Station zeigte sich als vollkommen genügend, um diese wenigstens bei Arbeit in Wasser von einem Salzgehalt bis $10 \%$ zu beseitigen ${ }^{1}$ ). Polarisation kann indessen durch Anwendung eines Wechselstromes vermieden werden.

Die Rubrizierung des Stromes in mehr als 16 Richtungen wird natürlich durch Vermehrung der Anzahl der Kontaktstücke und durch Vergrösserung des Registrierungsapparats ermöglicht. Eine andere Anordnung könnte die Anzahl der Richtungen zum dreifachen der Kontaktstücke an der Ebonitplatte vermehren. Für 8 Kontaktstücke wäre folgende Anordnung zu machen: 2 Wagen mit $15^{\circ}$ oder $1 / 24$ der Zirkelperipherie zwischen den Tangierungspunkten der Räder mit den Kontakten gleiten, wie früher erwähnt worden ist, ihre Mitten in einer Entfernung von $120^{\circ}$ voneinander, längs dem von den Kontaktstücken gebildeten Zirkel. Während der Strommesser sich einmal herumwendet, würden zum Beispiel folgende Kontaktstücke berührt werden und also die zugehörigen Federn zugleich schreiben, nämlich Nr. $8,1,3 ; 1,3,4 ; 1,4 ; 1,2,4 ; 2,4,5$ u.s. w. wobei jede Kombination dem Strome von einem bestimmten Sektor von $15^{\circ}$ entspricht. Durch Schätzung kann noch grössere Genauigkeit erlangt werden.

Es ist zu beachten, dass beim Schlagzählerkontakte, während der Strom unterbrochen ist, die Entfernung zwischen seinen beiden Polen möglichst gross sei, auch sollten am liebsten diese Kontaktstücke aus Platina sein.

Eine von aussen schliessbare Zweigleitung zu der Leitungsbahn der ersten Feder ermöglicht es, auf dem Registrierungspapiere Zeichen zu machen, zum Beispiel für die Augenblicke, wo es versucht wird, die Lage des Schiffes im Verhältnis zu einem fixen Punkte zu bestimmen.

Eine Veränderung, die ohne Zweifel zur leichteren Handtierung des Apparats beitragen würde, wäre es, die zwei Aufhängungsdrähte durch zwei Kabel zu ersetzen, von denen jedes die halbe Anzahl der Leitungsbahnen enthält.

Es ist selbstverständlich, dass die Isolierung innerhalb des Apparats sehr sorgfältig sein muss.

Schliesslich ist vielleicht noch hinzuzufügen, dass der Quecksilber-

1) Das als Anode verwandte $\operatorname{Rad} H$ ist nunmehr mit einer dünnen Platinplatte am Kontaktorte versehen, wodurch die Wirkung der Polarisation bei der Novemberterminfahrt eine sehr kleine wurde. (Nachträgliche Bemerkung.) 


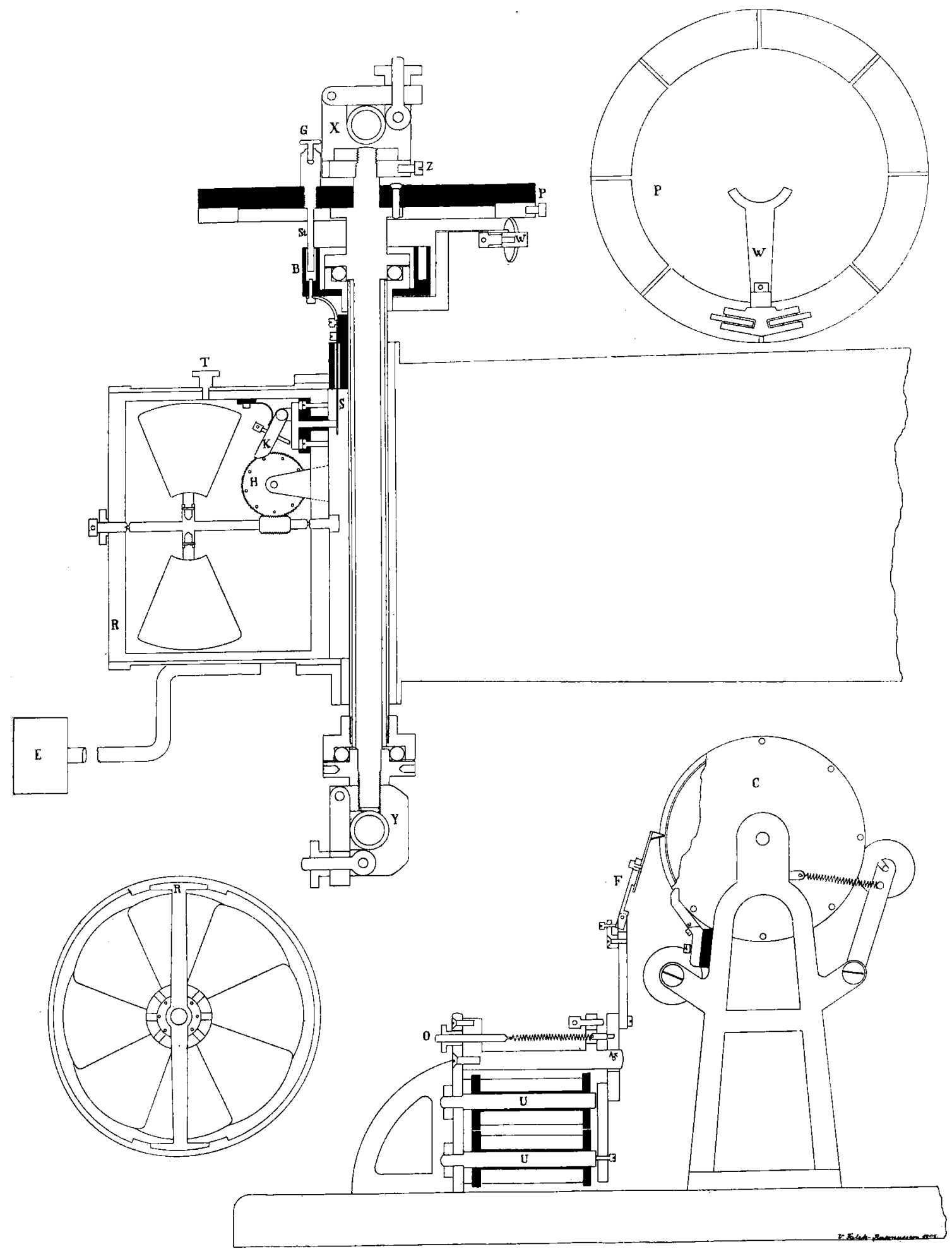

Strommesser von Rolf J. Wirtiva ( $1 / 8$ nat. Grösse) 
behälter tief genug sein muss, um das Ausrinnen des Quecksilbers bei einem etwaigen Stosse gegen das Schiff zu verhüten.

Dem Präsidenten der Finnischen Hydrographisch-Biologischen Kommission, Herrn Professor Anders Donner, möchte ich bei dieser Gelegenheit meine grosse Dankbarkeit aussprechen für sein persönliches Eingreifen, wodurch der Bau des oben beschriebenen Apparats ermöglicht wurde zu einer Zeit, als die Mittel der Kommission es nicht zugelassen hätten. Herrn Direktor E. BIESE bin ich gleichfalls vielen Dank schuldig für die Auskünfte, die ich von ihm erhalten habe, und für seinen wertvollen Rat hinsichtlich der Konstruktion registrierender Apparate. Herrn Mag. Phil. Sigurd Stenius, mit dem ich den Apparat betreffende Detailfragen diskutiert habe, bringe ich auch hier meinen Dank dar.

Nachtrag. Der oben beschriebene Apparat stellt sich ziemlich kostspielig. Durch folgende Abänderung wird er einfacher und kann beinahe ebensoviel leisten. Der elektromagnetische Registrierungsapparat wird durch zwei Ampèremesser ersetzt. Durch den einen wird die Schlagzählerleitung geführt, und mittels Sekundometer wird die Zeit zwischen zwei Kontakten, resp. 10 Flügelschlägen, beobachtet. Durch den anderen Ampèremesser geht ein Zweig der Zufuhrleitung. Dieser Zweig wird successiv mit den Leitungen von den Metallstïcken auf der Ebonitplatte $P$ verbunden, und der Ampèremesser gibt also an, welche Leitungen vom Wagen $W$ am Strommesser geschlossen sind. Am einfachsten kann dies wohl untersucht werden, wenn man auf dem arbeitenden Schiffe eine ähnliche Ebonitplatte hat, zu deren Metallstücken die Leitungen von denen der Platte $P$ geführt sind, während der Zweig der Zufuhrleitung nach dem Gang durch den Ampèremesser zu einem Rad geführt wird, das an einem radial gestellten beweglichen Arme befestigt längs der von den Metallstücken gebildeten Zirkelperipherie gleitet.

In dieser vereinfachten Gestalt wird der Strommesser wohl für Beobachtungen auf Feuerschiffen verwendbar sein. Die Handhabung erfordert zwei Personen, wenn aber, wie in dem folgenden Aufsatz erwähnt wird, den Schiffsbewegungen Rechnung getragen werden soll, sind wenigstens drei Personen nötig. 